\title{
Robust Image Registration with Global Intensity Transformation
}

\author{
Susana Ruano*, Guillermo Gallego*, Anthony Yezzi ${ }^{\dagger}$, Carlos Cuevas* and Narciso García* \\ * Grupo de Tratamiento de Imágenes (GTI), Univ. Politécnica de Madrid (UPM), Madrid, Spain. \\ ${ }^{\dagger}$ School of Electrical and Computer Engineering, Georgia Institute of Technology, Atlanta (GA), USA. \\ \{srs,ggb,ccr,narciso\}@gti.ssr.upm.es, ayezzi@ece.gatech.edu
}

\begin{abstract}
This paper presents a registration method for images with global illumination variations. The method is based on a joint iterative optimization (geometric and photometric) of the $L_{1}$ norm of the intensity error. Two strategies are compared to directly find the appropriate intensity transformation within each iteration: histogram specification and the solution obtained by analyzing the necessary optimality conditions. Such strategies reduce the search space of the joint optimization to that of the geometric transformation between the images.
\end{abstract}

Keywords-image registration; illumination transformations; first variation, histogram specification; aerial imaging.

\section{INTRODUCTION}

Image registration is the process of aligning two or more images and it is used as a preprocessing step in many areas such as medical imaging, augmented reality and remote sensing. Despite being a classic problem, research on registration under illumination variations due to acquisition with different sensors or at different times of the day can be found in recent literature [1].

There are two main approaches to estimate the geometric registration parameters: feature-based and area-based. Featurebased methods provide a layer of abstraction on top of the image intensities and are widely used, but they rely on the identification of enough distinctive features. When this is not possible, area-based (i.e., "direct" or "dense") methods that work on pixel intensities are preferred. These methods are usually based on the minimization of the $L_{2}$ intensity error norm, but this measure is not robust in presence of outliers [2].

Some of the approaches that take into account illumination variations try to jointly optimize both geometric and photometric parameters, thus increasing the size of the parameter space [3]. In the context of aerial imaging, some solutions deal with this problem by applying direct histogram specification but with a feature-based approach [4].

In this paper, a registration technique to deal with global illumination changes is presented. It is an area-based method that also considers a photometric transformation between images and minimizes the $L_{1}$ intensity error norm. To reduce the number of parameters in the iterative minimization process, two techniques that directly compute an intensity transformation are tested: histogram specification and a transformation

This work has been partially supported by the Ministerio de Economía y Competitividad of the Spanish Government under projects TEC2010-20412 (Enhanced 3DTV), TEC2013-48453 (MR-UHDTV), by AIRBUS under the project SAVIER, and by Consejo Social of the UPM.

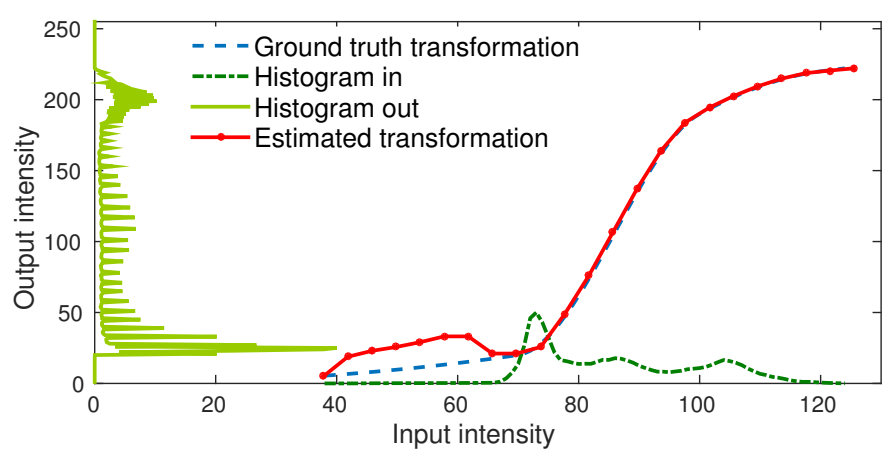

Fig. 1. Our method (red curve) can recover the global intensity transformation (blue curve) applied to the sensed image (for meaningful intensity levels in the histogram of the sensed image). The histograms of the sensed and transformed images are also displayed.

that satisfies the necessary optimality conditions. The technique is exemplified on aerial imagery, but it can also be applied to other types of images.

\section{Proposed Method}

\section{A. Joint optimization of geometric and photometric warpings}

Let $I_{1}$ be a target image and let $I_{2}$ be a sensed image that is to be registered with respect to $I_{1}$. The proposed method is based on the minimization of the energy functional

$$
E(g, f)=\left\|I_{1}-\hat{I}_{1}\right\|_{L_{1}\left(\Omega_{1}\right)},
$$

where $\|\cdot\|_{L_{1}\left(\Omega_{1}\right)}$ is the $L_{1}$ norm over the domain $\Omega_{1}$ of the target image, and $\hat{I}_{1}$ is the predicted image, which is obtained by applying a geometric transformation $g$ (defined by a finite number of parameters) and a global illumination change $f$ to the sensed image: $\hat{I}_{1}=f \circ I_{2} \circ g$, by function composition.

To jointly optimize (1) with respect to $g$ and $f$, we design a nested strategy: an iterative quasi-Newton method is used in a coarse-to-fine approach to evolve the parameters of the geometric transformation $g$ while, for each candidate $g$, a direct solver provides the intensity transformation $f$. Derivatives in the quasi-Newton method are approximated by finite differences. An initialization of the geometric parameters is required to start the algorithm. Next, we comment on two direct solvers for $f$.

\section{B. Histogram specification}

Histogram specification is a technique commonly used in image enhancement [5]. It is a generalization of the histogram 
equalization method but instead of producing an image with a uniform histogram it seeks to produce an output image with a specified histogram. We use this technique to compute the intensity transformation $f$ that gives the predicted image $\hat{I}_{1}$. To do that, the sensed image is warped $\left(I_{2} \circ g\right)$ according to the registration parameters and its histogram is transformed to match that of the target image $I_{1}$. This allows for a comparison between intensity levels of the target and predicted images.

\section{Intensity transformation satisfying the optimality condition}

The histogram specification technique is not aware of the pixel location in the image and is not obtained from (1). Our goal is to find an appropriate $f$ regarding these considerations. Consequently, we analyze the first variation of (1) to find a transformation $f$ that satisfies the necessary optimality condition (i.e., zero functional gradient: $\delta E / \delta f=0$ ).

After some calculations in the Euler-Lagrange equations, and considering the intensity level sets of the images, we obtain the functional gradient of (1):

$$
\frac{\delta E}{\delta f}(\mu)=\int_{\Omega_{1}} \operatorname{sgn}\left(f(\mu)-I_{1}(\mathbf{x})\right) \delta\left(\mu-\left(I_{2} \circ g\right)(\mathbf{x})\right) d \mathbf{x}
$$

where $\operatorname{sgn}(\cdot)$ is the sign function and $\delta(\cdot)$ is the Dirac delta.

The optimality condition depends on the level sets of $I_{2} \circ g$. Further analysis of setting (2) to zero reveals the direct solver: for each intensity level $\mu$ we can find the corresponding $f(\mu)$ by computing the median of the intensity values of the target image for those $\mathbf{x}$ in the level sets of $I_{2} \circ g$, i.e., $\mu=\left(I_{2} \circ g\right)(\mathbf{x})$. Fig. 1 shows the recovered $f$ using this method, which does not need to be monotonic and provides a good fit in the input intensity levels with significant histogram mass.

\section{EXPERIMENTS AND RESULTS}

To evaluate the proposed method we have used multiple pairs of aerial images containing critical situations (e.g., poor textures or large illumination changes). All of them have been acquired using the same procedure as in [6] to simulate an Unmanned Aerial Vehicle (UAV) flying at altitudes between 6 and $10 \mathrm{~km}$ and using a downward-looking camera. For simplification, to test both intensity transformation strategies in II-B and II-C, the geometric warping applied to the images is limited to a rotation. In the performed experiments we have tested two types of illumination changes: (1) slight intensity changes due to the use of different camera sensor parameters (gain, shutter speed, etc.) and (2) natural illumination changes (e.g., day or night). The former is simulated by applying an arbitrary (e.g., linear) transformation and the latter is done by changing the acquisition settings.

Fig. 2 illustrates some representative results comparing the estimations obtained by the coarse-to-fine nested optimization strategy in II-A using either histogram specification (method in II-B) or the transformation that fits the necessary optimality conditions (method in II-C). The first row (Fig. 2(a)) shows the results of applying the registration method to an image of an urban area that is rich in texture. It can be observed that both methods provide an accurate registration in this situation. Fig. 2(b) depicts two images of a desert area, poor in texture. This example shows that the choice in II-C is more reliable

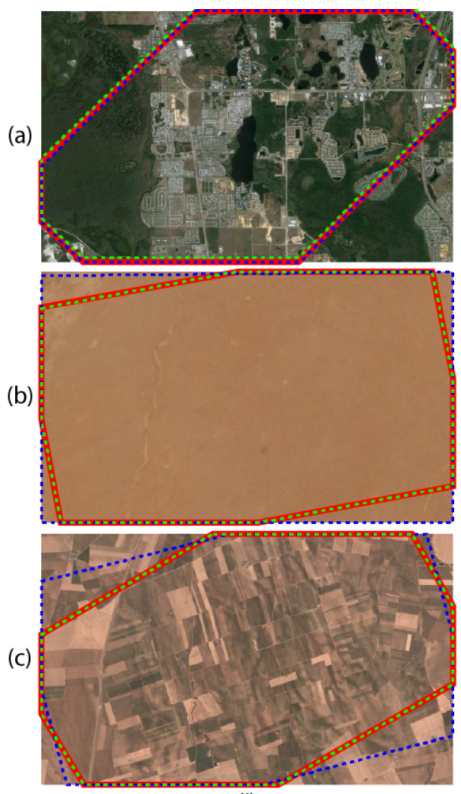

(i)
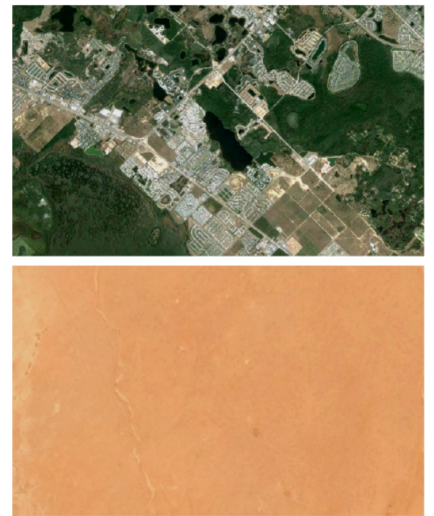

Fig. 2. Right column: sensed images. Left column: target images, with color notation: red (exact registration), blue (registration with II-B), green (registration with II-C).

than II-B in these situations because an accurate registration is obtained whereas it is not with the later. Finally, in Fig. 2(c) a scenario of two images with natural illumination changes is shown. In this case, the method II-C is also the only one that achieves an accurate registration.

\section{CONCLUSION}

We have proposed an image registration method that can also deal with global illumination changes. It is based on a joint optimization of an energy functional on the $L_{1}$ norm. In our nested iterative approach, two strategies to recover the illumination transformation for a given geometric warping are presented. The results show a better performance of the registration by means of the illumination transformation that is based on the optimality condition analysis rather than that of the histogram specification technique.

\section{REFERENCES}

[1] M. Fouad, R. Dansereau, and A. Whitehead, "Image Registration Under Illumination Variations Using Region-Based Confidence Weighted MEstimators," IEEE Trans. Image Process., vol. 21, no. 3, pp. 1046-1060, 2012.

[2] B. Zitov and J. Flusser, "Image registration methods: a survey," Image and Vision Computing, vol. 21, no. 11, pp. 977-1000, 2003.

[3] R. Frenkel and J. Francos, "Registration of geometric deformations in the presence of varying illumination," in IEEE Int. Conf. Image Process. (ICIP), vol. 3, 2007, pp. III: 489-492.

[4] H. Tuo, L. Zhang, and Y. Liu, "Multisensor aerial image registration using direct histogram specification," in IEEE Int. Conf. Networking, Sensing and Control, vol. 2, 2004, pp. 807-812.

[5] R. Gonzalez and R. Woods, Digital Image Processing. Prentice Hall, 2008.

[6] S. Ruano, G. Gallego, C. Cuevas, and N. García, "Aerial video georegistration using terrain models from dense and coherent stereo matching," in SPIE Int. Conf. Motion Imagery for ISR and Situational Awareness II, vol. 9089, 2014, pp. 90890V-1-10. 